\title{
CARACTERIZAÇÃO FÍSICO-QUÍMICA DO FÁRMACO ANTICHAGÁSICO BENZNIDAZOL
}

\author{
Flávia Pires Maximiano, Guilherme Hideki Yoshizane Costa e Jacqueline de Souza \\ Escola de Farmácia, Universidade Federal de Ouro Preto, Rua Costa Sena, 171, 35400-000 Ouro Preto - MG, Brasil \\ Marcílio Sérgio Soares da Cunha-Filho* \\ Instituto de Ciências da Saúde, Universidade Federal do Mato Grosso, Campus Universitário de Sinop, Av. Alexandre Ferronato, \\ 1200, 78557-267 Sinop - MT, Brasil
}

Recebido em 5/1/10; aceito em 18/4/10; publicado na web em 9/8/10

\begin{abstract}
PHYSICOCHEMICAL CHARACTERIZATION OF ANTICHAGASIC BENZNIDAZOLE. Currently, benznidazole (BNZ) is a unique therapeutic alternative available in Brazil to treat Chagas disease. Despite its traditional medical use, little is known about the chemical nature of this drug. A detailed study of the physicochemical properties of BNZ was performed using multiple assays. Thermal, diffractometric, morphological and reological drug profiles were obtained. The partition coefficient and solubility results allowed this drug to be classified as a class IV drug according to the biopharmaceutical classification system. This information will be useful for the development of more effective BNZ formulations and for establishing the quality profile of BNZ.
\end{abstract}

Keywords: benznidazole; preformulation; biopharmaceutical classification.

\section{INTRODUÇÃO}

A pesquisa e o desenvolvimento de medicamentos inovadores têm sido uma empreitada cada vez mais cara e complexa, o que torna mais escassa a introdução de novas moléculas no mercado. ${ }^{1}$ Estima-se que de cada 30.000 compostos sintetizados, apenas $0,003 \%$ chegam a se tornar fármacos disponíveis no comércio. ${ }^{2}$ Dentre as causas que explicam estes inúmeros fracassos estão problemas derivados de sua biodisponibilidade e toxicidade, que podem estar relacionados ao escasso conhecimento acerca da natureza da molécula em estudo. ${ }^{3}$

Neste contexto, os estudos de pré-formulação, realizados nas etapas de pesquisa pré-clínica e clínica de fármacos inovadores, e que visam acumular o máximo de informação sobre o comportamento das novas entidades químicas, constituem um valioso instrumento para traçar estratégias de desenvolvimento racional de formulações mais eficazes e seguras e, portanto, com maiores possibilidades de êxito. ${ }^{4,5}$ Diversos aspectos concernentes à substância ativa, como a biodisponibilidade a partir da forma farmacêutica, ou ao medicamento, como seu prazo de validade, e até mesmo seu processamento industrial são afetados pelas propriedades físico-químicas dos fármacos.

O Benznidazol [BNZ] (N-benzyl-2-nitro-1-imidazole-acetamide) (Figura 1), constitui um dos únicos recursos terapêuticos disponíveis no combate à doença de Chagas há décadas. Este medicamento é bastante efetivo na fase aguda da doença, mas existem relatos de baixa ou nenhuma eficácia na fase crônica. Efeitos secundários severos associados à utilização desse fármaco têm sido reportados, tendo como consequência, a interrupção do tratamento medicamentoso. ${ }^{6}$<smiles>O=C(Cn1ccnc1[N+](=O)[O-])NCc1ccccc1</smiles>

Figura 1. Estrutura molecular de BNZ
Apesar de ser um fármaco já consolidado no mercado e bastante estudado do ponto de vista farmacológico, as propriedades físicoquímicas do BNZ são praticamente desconhecidas. Estudos de préformulação poderiam propiciar uma maior compreensão das suas características biofarmacêuticas e permitir vislumbrar alternativas para melhorar sua ação terapêutica.

Desta forma, o objetivo deste estudo foi traçar o perfil físicoquímico do BNZ através de diferentes ensaios de caracterização, como forma de estabelecer parâmetros de qualidade para este composto e auxiliar no melhoramento tecnológico de medicamentos à base deste antichagásico.

\section{PARTE EXPERIMENTAL}

\section{Materiais}

Benznidazol [BNZ] (MW 260,25) produzido pelos laboratórios Roche, lote 13871. Todos os solventes e reagentes utilizados nos ensaios foram de pureza analítica.

\section{Solubilidade}

Os estudos foram conduzidos utilizando excesso de fármaco em $10 \mathrm{~mL}$ de diferentes meios líquidos. As amostras pulverizadas de BNZ foram inicialmente submetidas a ultrassom por $15 \mathrm{~min}$ e colocadas sob agitação magnética vigorosa a $25{ }^{\circ} \mathrm{C}\left( \pm 2{ }^{\circ} \mathrm{C}\right)$ até o estabelecimento do equilíbrio, a seguir foram filtradas $(0,45 \mu \mathrm{m})$ e doseadas por metodologia espectrofotométrica previamente validada a $324 \mathrm{~nm}$. Este experimento foi realizado em triplicata e a saturação das amostras foi previamente determinada em $48 \mathrm{~h}$.

Meios líquidos de diferentes naturezas e polaridades foram utilizados como solventes no ensaio de solubilidade e encontram-se listados a seguir: água, metanol, etanol, acetona, acetato de etila; isopropanol, diclorometano, hexano, clorofórmio, éter de petróleo,

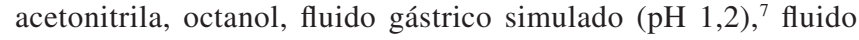
entérico simulado (pH 6,8), ${ }^{7}$ solução aquosa de lauril sulfato de sódio [LSS] em diferentes concentrações $(0,1 ; 0,2 ; 0,5$ e $1,0 \%)$, óleo mineral e óleo de amêndoas. 


\section{Velocidade intrínseca de dissolução [VID]}

Para determinar a velocidade intrínseca de dissolução do BNZ, foram preparados discos compactos não desintegráveis deste fármaco, empregando pressões elevadas em uma compressora excêntrica Fabbe ${ }^{\circledR}$ utilizando punções circulares planas de $12 \mathrm{~mm}$. Os comprimidos de BNZ obtidos com elevada dureza e superfícies lisas foram recobertos de parafina, de forma que o topo e as laterais do comprimido ficassem revestidos e somente uma das faces de área calculada em $1,13 \mathrm{~cm}^{2}$ permanecesse exposta.

O ensaio de dissolução foi realizado em um dissolutor Nova Etica $^{\circledR}$, utilizando $900 \mathrm{~mL}$ de fluido gástrico simulado como meio de dissolução a $37{ }^{\circ} \mathrm{C}$ e pás como aparato de agitação a uma velocidade de $75 \mathrm{rpm} .^{7}$ Os comprimidos foram fixados ao fundo da cubeta com auxílio de uma malha de aço inox e com a face sem parafina exposta ao meio de dissolução. Amostras do meio foram coletadas em tempos programados, filtradas e quantificadas quanto à concentração de BNZ, utilizando metodologia espectrofotométrica previamente validada. $\mathrm{O}$ ensaio foi realizado em quintuplicata e a VID foi obtida a partir do coeficiente angular da regressão linear da quantidade de fármaco dissolvido por superfície em função do tempo, conforme descreve a equação de Noyes-Nernst. ${ }^{8}$

\section{Coeficiente de partição pelo método de agitação moderada}

O coeficiente de partição do BNZ foi obtido conforme método de agitação moderada validado para fármacos de baixa solubilidade aquosa. ${ }^{9}$ Utilizou-se como fase orgânica 1-octanol e como fase aquosa, água destilada, fluido gástrico simulado $\mathrm{pH} 1,2$ e fluido entérico simulado $\mathrm{pH}$ 6,8.7 Os meios de partição foram submetidos à saturação mútua por 2 dias, com temperatura controlada de $25^{\circ} \mathrm{C}$ antes do estudo.

$\mathrm{O}$ BNZ foi previamente solubilizado na fase orgânica e colocado junto à fase aquosa sob agitação magnética durante 5 dias, a $25^{\circ} \mathrm{C}$. A turbulência na interface de separação dos meios foi controlada, de forma a evitar a emulsificação do octanol na fase aquosa. Ao final, as concentrações de fármaco nas fases aquosa e octanólica foram quantificadas por espectrofotometria na região do ultravioleta a $324 \mathrm{~nm}$, utilizando-se metodologia validada. O experimento foi realizado em triplicata. O coeficiente de partição foi representado pelo logaritmo de base 10 da relação entre a concentração de fármaco nas fases octanólica e aquosa $\left[\log \mathrm{P}_{\mathrm{ow}}\right]$.

\section{Perfil térmico}

De forma a determinar o comportamento térmico deste produto, medidas de calorimetria diferencial de varredura [DSC] foram conseguidas utilizando-se os calorímetros MDSC Q100 (TA Instruments ${ }^{\circledR}$ ) e DSC 2010 (TA Instruments ${ }^{\circledR}$ ). Nitrogênio foi utilizado como gás de purga com fluxo de $50 \mathrm{~mL} / \mathrm{min}$. A calibração do equipamento foi realizada com padrões de índio e zinco.

Amostras de BNZ, de aproximadamente $3 \mathrm{mg}$, foram colocadas em cápsulas de alumínio planas tapadas e submetidas à velocidade de aquecimento de $10{ }^{\circ} \mathrm{C} / \mathrm{min}$ no intervalo de temperatura de 30 a 300 ${ }^{\circ} \mathrm{C}$. Ciclos de aquecimento-resfriamento-aquecimento também foram realizados de forma a confirmar os fenômenos térmicos empregando velocidades de $10{ }^{\circ} \mathrm{C} / \mathrm{min}$.

A pureza do princípio ativo foi conseguida a partir de análises segundo a equação de van't Hoff, empregando-se o software TA Universal analysis $2000 \mathrm{~V} 4$. Para tanto, amostras de $2 \mathrm{mg}$ foram submetidas a aquecimento de $2^{\circ} \mathrm{C} / \mathrm{min}$, partindo-se da temperatura de 30 até $200^{\circ} \mathrm{C}$.

A umidade residual do fármaco foi determinada utilizando termobalança de infravermelho Eurotherm (Gibertini $\left.{ }^{\circledR}\right)$. Foram pesadas amostras contendo $1,0 \mathrm{~g}$ de fármaco e submetidas a aquecimento através do infravermelho, até que a variação de peso fosse inferior a $0,25 \% \mathrm{p} / \mathrm{p}$. Os valores representam a média de 5 determinações e são expressos em porcentagem (\%p/p).

\section{Perfil difractométrico}

Ensaios de difração de raios-X do pó foram realizados em amostras de BNZ empregando-se um equipamento XRD-6000 $\left(\right.$ Shimadzu $\left.^{\circledR}\right)$, utilizando monocromador de grafite e ânodo de Fe no intervalo de 2 a $60^{\circ} 2 \theta$.

\section{Superfície específica}

A área superficial específica do BNZ foi determinada por meio da técnica de adsorção de nitrogênio aplicando-se o modelo proposto por Brunauer, Emmett e Teller [BET]. ${ }^{10,11}$ Amostras de fármaco previamente degaseificadas por $24 \mathrm{~h}$ a $40{ }^{\circ} \mathrm{C}$ foram analisadas no equipamento BET Surface Area Analyser Nova ${ }^{\circledR} 1000$.

\section{Densidade real}

A densidade real do fármaco foi determinada por picnometria de hélio, conforme descrito na Farmacopeia Americana, em duplicata, utilizando um picnômetro Quantachrome MPY. ${ }^{7}$

\section{Análise granulométrica e morfológica}

A avaliação da morfologia dos cristais de BNZ foi realizada por microscopia eletrônica de varredura (MEV) utilizando-se um microscópio LEO-435VP. As amostras foram previamente metalizadas com ouro. A distribuição dos tamanhos de partícula do lote comercial de BNZ foi determinada por tamisação a partir de $50 \mathrm{~g}$ de material utilizando um tamisador Bertel ${ }^{\circledR}$. O tamanho médio de partícula do fármaco foi calculado a partir de análise estatística utilizando-se o método de probabilidades. ${ }^{12}$

\section{Propriedades de fluxo}

Medidas reológicas dos cristais de BNZ foram avaliadas baseandose nas técnicas propostas por Carr e em parâmetros farmacopeicos. ${ }^{7,13}$

$\mathrm{O}$ ângulo de repouso foi medido pelo cone de pó formado pelo escoamento do fármaco através de um funil de dimensões padronizadas sobre uma superfície plana. $\mathrm{O}$ ângulo formado entre o cone e a superfície foi medido com auxílio de um transferidor. O tempo de escoamento foi determinado pela medida do tempo necessário para o escoamento de uma quantidade pré-definida de fármaco através de um funil padronizado, utilizando-se um cronômetro digital. A compressibilidade e o índice de Hausner foram calculados a partir de medidas de volume aparente do sólido antes $\left[\mathrm{V}_{0}\right]$ e após $\left[\mathrm{V}_{\mathrm{f}}\right]$ ser submetido a compactações com auxílio de uma proveta empregando a seguintes equações:

$$
\begin{gathered}
\text { Compressibilidade }=\left(\mathrm{V}_{0}-\mathrm{V}_{\mathrm{f}}\right) / \mathrm{V}_{0} * 100 \\
\text { Índice de Hausner }=\mathrm{V}_{0} / \mathrm{V}_{\mathrm{f}}
\end{gathered}
$$

A densidade de enchimento foi calculada a partir da relação entre peso de BNZ e volume ocupado pelo sólido após escoamento espontâneo para uma proveta. Todas as medidas de fluxo de pó foram realizadas em triplicata.

\section{Higroscopicidade}

A habilidade do BNZ em interagir com a umidade ambiental atmosférica foi estudada empregando-se o modelo experimental estabelecido 
por Callahan e colaboradores. ${ }^{14}$ Amostras de BNZ foram expostas a 12 condições de umidade relativa diferentes (5-98\%), durante 7 dias. Estas condições foram obtidas utilizando-se soluções salinas saturadas em sistemas fechados a $25^{\circ} \mathrm{C} .{ }^{15}$ A quantidade de água absorvida pelas amostras foi medida por gravimetria através do monitoramento do incremento de peso percentual das amostras de BNZ em pó, previamente dessecadas, e após o tempo de exposição nas diferentes condições de umidade relativa atmosférica. $\mathrm{O}$ experimento foi executado em triplicata.

\section{RESULTADOS E DISCUSSÃO}

\section{Solubilidade}

A solubilidade dos fármacos é uma propriedade que influencia diferentes aspectos relativos à farmacocinética e estabilidade química da molécula. Auxilia também na escolha do solvente mais adequado para utilizações analíticas, assim como na eleição do veículo mais idôneo para uso em ensaios in vivo ou para uma possível formulação líquida do fármaco.

Os solventes com maior capacidade para dissolver o BNZ e que, portanto, são os mais recomendáveis para utilização em métodos analíticos e extrações deste composto foram o metanol, a acetonitrila e a acetona (Tabela 1).

Notam-se algumas diferenças dos resultados deste ensaio (Tabela 1) com respeito aos valores de solubilidade descritos na literatura. A solubilidade aquosa do BNZ é citada em algumas publicações como $0,4 \mathrm{mg} / \mathrm{mL},{ }^{16}$ afastando-se consideravelmente do valor experimental encontrado de $0,2 \mathrm{mg} / \mathrm{mL}$ em água destilada ou nos fluidos gástrico e entérico simulados. Este dado demonstra que o fármaco não se ioniza nas condições avaliadas, o que já era esperado, uma vez que a molécula de BNZ não apresenta grupos facilmente ionizáveis (Figura 1).

Observa-se também que mesmo apresentado uma solubilidade limitada em água, os valores encontrados para alguns veículos oleosos (óleo de amêndoas e óleo mineral) são ainda mais reduzidos. A presença de um tensoativo (Lauril sulfato de sódio) possibilita incrementar de forma importante a solubilidade aquosa do BNZ somente em concentrações elevadas (Tabela 1).

Destacam-se ainda as divergências existentes em relação à classificação da solubilidade farmacopeica e a monografia do BNZ matéria-prima encontrada na Farmacopeia Brasileira IV ed. ${ }^{17}$ Para alguns solventes (acetona e isopropanol), a solubilidade experimental é superior à descrita na monografia, o que pode ser explicado pela diferença de metodologia empregada, uma vez que o ensaio farmacopeico não garante condições de saturação das amostras. No entanto, para os solventes etanol, acetato de etila, diclorometano e hexano as solubilidades farmacopeicas declaradas são superiores aos dados de solubilidade determinados neste estudo. Os resultados aportados poderão servir de base para uma futura revisão e complementação da monografia oficial do BNZ na Farmacopeia Brasileira.

\section{Velocidade intrínseca de dissolução [VID]}

A velocidade intrínseca de dissolução [VID] é uma característica inerente a cada substância sólida e, diferentemente das medidas de solubilidade, que se baseiam em equilíbrio, esta constitui um parâmetro cinético dependente da molhabilidade e da difusibilidade do composto. A determinação da VID fornece indícios se determinado fármaco apresentará problemas de biodisponibilidade em função de suas características de dissolução.

Durante o ensaio, a quantidade de BNZ dissolvido no meio aumenta com o tempo de forma linear até os primeiros $20 \mathrm{~min}$, quando a velocidade de dissolução parcial do fármaco começa a declinar,
Tabela 1. Dados de solubilidade médios de BNZ em diferentes solventes a $25^{\circ} \mathrm{C}$. Entre parênteses está representado o desvio padrão dos resultados

\begin{tabular}{|c|c|c|c|c|}
\hline \multicolumn{2}{|c|}{ Solvente } & $\begin{array}{l}\text { Solubilidade } \\
(\mathrm{mg} / \mathrm{mL})\end{array}$ & $\begin{array}{l}\text { Classificação experi- } \\
\text { mental }^{\mathrm{a}}\end{array}$ & $\begin{array}{c}\text { Monografia } \\
\text { Farm. Bras. IV }\end{array}$ \\
\hline \multicolumn{2}{|c|}{ Água Destilada } & $0,237(0,012)$ & Muito pouco solúvel & $\begin{array}{l}\text { Muito pouco } \\
\text { solúvel }\end{array}$ \\
\hline \multicolumn{2}{|c|}{ Metanol } & $13,078(2,240)$ & Ligeiramente solúvel & $\begin{array}{l}\text { Ligeiramente } \\
\text { solúvel }\end{array}$ \\
\hline \multicolumn{2}{|c|}{ Etanol } & $4,989(0,359)$ & Pouco solúvel & $\begin{array}{l}\text { Ligeiramente } \\
\text { solúvel }\end{array}$ \\
\hline \multicolumn{2}{|c|}{ Acetona } & $30,546(0,249)$ & Ligeiramente solúvel & Pouco solúvel \\
\hline \multicolumn{2}{|c|}{ Acetado de etila } & $3,550(0,092)$ & Muito pouco solúvel & $\begin{array}{l}\text { Ligeiramente } \\
\text { solúvel }\end{array}$ \\
\hline \multicolumn{2}{|c|}{ Isopropanol } & $1,940(0,130)$ & Pouco solúvel & $\begin{array}{l}\text { Muito pouco } \\
\text { solúvel }\end{array}$ \\
\hline \multicolumn{2}{|c|}{ Diclorometano } & $3,138(0,192)$ & Pouco solúvel & $\begin{array}{l}\text { Ligeiramente } \\
\text { solúvel }\end{array}$ \\
\hline \multicolumn{2}{|c|}{ Hexano } & $0,141(0,048)$ & Muito pouco solúvel & Solúvel \\
\hline \multicolumn{2}{|c|}{ Clorofórmio } & $1,499(0,267)$ & Muito pouco solúvel & $\begin{array}{l}\text { Muito pouco } \\
\text { solúvel }\end{array}$ \\
\hline \multicolumn{2}{|c|}{ Éter de petróleo } & $0,067(0,007)$ & Praticamente insolúvel & $\begin{array}{l}\text { Praticamente } \\
\text { insolúvel }\end{array}$ \\
\hline \multicolumn{2}{|c|}{ Acetonitrila } & $24,741(2,866)$ & Ligeiramente solúvel & - \\
\hline \multicolumn{2}{|c|}{ Octanol } & $0,530(0,024)$ & Muito pouco solúvel & - \\
\hline \multicolumn{2}{|c|}{ Fluido gástrico } & $0,236(0,004)$ & Muito pouco solúvel & - \\
\hline \multicolumn{2}{|c|}{ Fluido entérico } & $0,244(0,012)$ & Muito pouco solúvel & - \\
\hline \multirow[t]{4}{*}{ LSS } & $0,1 \%$ & $0,219(0,002)$ & Muito pouco solúvel & - \\
\hline & $0,2 \%$ & $0,229(0,014)$ & Muito pouco solúvel & - \\
\hline & $0,5 \%$ & $0,299(0,008)$ & Muito pouco solúvel & - \\
\hline & $1,0 \%$ & $0,406(0,013)$ & Muito pouco solúvel & - \\
\hline \multicolumn{2}{|c|}{ Óleo mineral } & $0,003(0,001)$ & Praticamente insolúvel & - \\
\hline \multicolumn{2}{|c|}{ Óleo de amêndoas } & $0,029(0,001)$ & Praticamente insolúvel & - \\
\hline
\end{tabular}

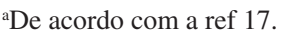

em função da variabilidade das condições hidrodinâmicas do ensaio (Figura 1S, material suplementar). A VID obtida para o BNZ, calculada a partir da porção linear inicial da curva, foi de $0,18 \mathrm{mg} / \mathrm{cm}^{2} /$ min, valor bastante inferior ao mínimo recomendável de $1 \mathrm{mg} / \mathrm{cm}^{2} /$ min, o que indica que a dissolução deste fármaco poderá ser um fator limitante de sua absorção. ${ }^{8}$

\section{Coeficiente de partição pelo método de agitação moderada}

O coeficiente de partição fornece informações sobre a facilidade dos fármacos em atravessar as membranas biológicas. Esta medida da permeabilidade in vitro foi determinada para o BNZ utilizandose o método de agitação moderada, que emprega uma agitação controlada na interface das fases orgânica e aquosa, de maneira a evitar a formação de microgotas de octanol na fase aquosa. ${ }^{9}$ Este método se apresenta mais confiável que o método tradicional de agitação em funil de separação, que promove a emulsificação da fase orgânica na fase aquosa propiciando uma sobre-estimação da concentração do fármaco nesta fase, que no caso de fármacos pouco solúveis em água, como o BNZ, acarreta um erro significativo para o cálculo de $\log \mathrm{P}_{\text {ow }}$.

Os valores de $\log _{\text {ow }}$ para o BNZ encontram-se descritos na Tabela 2. Valores similares de $\log \mathrm{P}_{\text {ow }}$ em torno a 0,7 , foram encontrados na literatura para os diferentes meios aquosos. Estimativas teóricas relativas ao seu $\log \mathrm{P}_{\text {ow }}$ utilizando modelagem molecular por diferentes softwares disponíveis (ClogP, KOWWIN, miLogP 2.2, ACD $\operatorname{LogP}$ ) apresentaram valores entre 0,7 a $1,2 .{ }^{18}$ Os dados experimentais 
situam-se dentro da faixa prevista in silico, no entanto, apresentam um amplo intervalo de valores dependendo do modelo matemático empregado pelos softwares, o que evidencia a necessidade de sua determinação experimental como forma de elevar o nível de precisão deste ensaio (Tabela 2).

Tabela 2. Coeficiente de partição de BNZ baseado em valores experimentais e de estimativas in silico. Entre parênteses está representado o desvio padrão dos resultados

\begin{tabular}{|c|c|c|c|c|c|c|}
\hline \multirow{2}{*}{$\begin{array}{l}\text { Meio } \\
\text { orgânico }\end{array}$} & \multirow{2}{*}{$\begin{array}{c}\text { Meio } \\
\text { aquoso }\end{array}$} & \multirow{2}{*}{$\log \mathrm{P}_{\text {ow }}$} & \multicolumn{4}{|c|}{$\begin{array}{c}\text { Estimativas matemáticas } \\
\log \mathrm{P}\end{array}$} \\
\hline & & & $\mathrm{Clog}^{\circledR}$ & KowWin $^{\circledR}$ & $\operatorname{miLog} \mathrm{P} 2.2^{\circledR}$ & $\mathrm{ACD} \log \mathrm{P}^{\circledR}$ \\
\hline 1-octanol & $\begin{array}{l}\text { Água } \\
\text { destilada }\end{array}$ & $\begin{array}{c}0,772 \\
(0,019)\end{array}$ & 0,9 & 1,22 & 0,778 & 0,910 \\
\hline 1-octanol & $\begin{array}{l}\text { Fluido } \\
\text { gástrico }\end{array}$ & $\begin{array}{c}0,784 \\
(0,011)\end{array}$ & & & & \\
\hline 1-octanol & $\begin{array}{c}\text { Fluido } \\
\text { entérico }\end{array}$ & $\begin{array}{c}0,763 \\
(0,027)\end{array}$ & & & & \\
\hline
\end{tabular}

O tradicional sistema de classificação biofarmacêutica divide os fármacos em 4 categorias, segundo suas características de solubilidade e de permeabilidade. ${ }^{19}$ A falta de informações experimentais sobre estas medidas para o BNZ tem causado interpretações dúbias sobre a classificação biofarmacêutica deste fármaco que, segundo publicações recentes, chegou a ser inserido na categoria II (baixa solubilidade e alta permeabilidade) e III (alta solubilidade e baixa permeabilidade). ${ }^{20,21}$

$\mathrm{O}$ valor de $\log \mathrm{P}_{\text {ow }}$ do $\mathrm{BNZ}$, embora relativamente alto, ainda não garante uma imediata permeação da molécula, que só ocorreria com substâncias com $\log \mathrm{P}_{\text {ow }}$ superiores a 1,7. ${ }^{21}$ Considerando esta medida de permeabilidade e a reduzida solubilidade aquosa do BNZ (Tabela 1), pode-se incluir este antichagásico no grupo IV da classificação biofarmacêutica, ou seja, um fármaco de baixa permeabilidade e de baixa solubilidade.

\section{Perfil térmico}

Um número elevado de eventos químicos e físicos pode ser detectado utilizando-se técnicas térmicas como o DSC. ${ }^{22}$ Conforme se observa na Figura 2, o BNZ apresenta um pico endotérmico de fusão a $191,3{ }^{\circ} \mathrm{C}$ com entalpia associada de aproximadamente 140 $\mathrm{J} / \mathrm{g}$ e degradação iniciando-se a partir de $240{ }^{\circ} \mathrm{C}$. A Figura 2 mostra também que o resfriamento do BNZ, logo após a fusão, promove a recristalização do fármaco, evidenciado por um pico exotérmico bem definido a $115^{\circ} \mathrm{C}$, envolvendo uma entalpia de $95 \mathrm{~J} / \mathrm{g}$. Durante o segundo aquecimento desta amostra, a fusão do fármaco ocorre novamente na mesma temperatura e com valor similar de energia, o que confirma a natureza destes fenômenos e comprova que a segunda cristalização origina a mesma fase cristalina inicial.

A determinação da pureza do BNZ possui especial relevância, uma vez que suas impurezas de síntese não estão estudadas a nível toxicológico. O método térmico baseado na equação de van't Hoff é recomendado pela Farmacopeia para análise de fármacos e representa um método rápido e preciso para este tipo de determinação. ${ }^{7} \mathrm{O}$ lote de BNZ analisado apresenta elevada pureza, estimada em 99,43\%. A umidade residual encontrada para o lote testado foi de $0,93 \%( \pm 0,38)$.

\section{Perfil difratométrico}

O difractograma de um composto é capaz de determinar sua pureza física. Neste ensaio foi estabelecido o padrão de difração do pó para o antichagásico BNZ. Observa-se um perfil tipicamente policristalino, com picos principais bem definidos posicionados a 9,$4 ; 13,9 ; 18,9 ; 20,4 ; 21,5$ e $26,1^{\circ} 2 \theta$ (Figura 3).

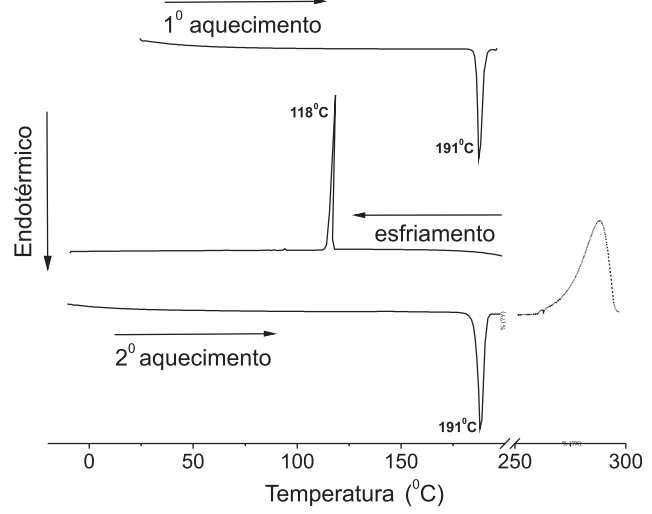

Figura 2. Perfil térmico de BNZ através de DSC. Termograma correspondente a um ciclo de aquecimento-esfriamento-aquecimento de $\mathrm{BNZ}\left(+200^{\circ} \mathrm{C} ;-20\right.$ ${ }^{\circ} \mathrm{C} ;+300^{\circ} \mathrm{C}$ )

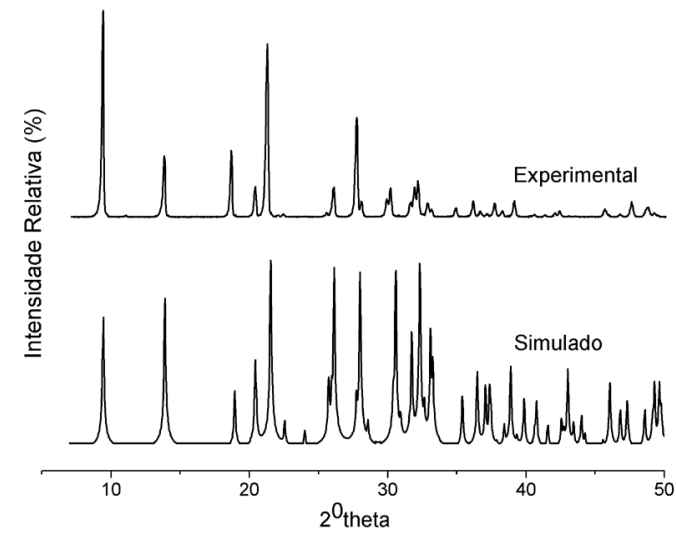

Figura 3. Difratograma de raios- $X$ do pó de BNZ experimental e simulado

Podem-se apreciar algumas flutuações nas intensidades relativas dos picos do BNZ quando comparado com seu difractograma padrão simulado a partir dos dados de raios-X do monocristal (Figura 3). ${ }^{23}$ Estas variações podem ser justificadas pela orientação preferencial dos cristais e indicam tratar-se da mesma forma cristalina estabelecida na literatura. Alterações na fase cristalina do fármaco, como o aparecimento de formas polimórficas, seriam facilmente identificadas por este teste, com um elevado grau de segurança. Devido à relevância do controle cristalino atualmente recomendado para fármacos, o perfil difratométrico constitui, portanto, uma ferramenta importante, juntamente com os métodos térmicos, para ser aplicada à rotina do controle de qualidade de matérias-primas de uso farmacêutico.

\section{Superfície específica}

A área superficial específica do BNZ, determinada pelas isotermas de adsorção do nitrogênio, revelou um valor de superfície de $0,5 \mathrm{~m}^{2} / \mathrm{g}$ e reduzida porosidade das partículas, estimada em $1,4 \times 10^{-3} \mathrm{~cm}^{3} / \mathrm{kg}$. Estes dados explicam sua lenta velocidade intrínseca de dissolução e justificam as deficientes propriedades de fluxo deste produto.

\section{Densidade real}

A densidade de um sólido pode adotar diferentes valores em função da técnica empregada em sua determinação. A densidade picnométria ou real encontrada para o BNZ foi de $1,35 \mathrm{~g} / \mathrm{cm}^{3}$. Este valor se aproxima do referenciado no ensaio do monocristal $(1,44$ $\mathrm{g} / \mathrm{cm}^{3}$ ), o que comprova a inexistência de microporos ou de espaços 
vazios impenetráveis pelo gás nos cristais de BNZ. ${ }^{23}$ Esta medida de densidade real poderá servir como referência para o controle do polimorfismo ou do grau de cristalinidade do BNZ, uma vez que alterações nestes parâmetros influenciam consideravelmente a densidade de um sólido.

\section{Análise granulométrica e morfológica}

Os cristais monoclínicos de BNZ, utilizados na produção industrial do medicamento comercial Rochagan ${ }^{\circledR}$, apresentam hábito cristalino acicular, conforme se aprecia na fotomicrografia da Figura $2 \mathrm{~S}$, material suplementar. A análise morfológica de fármacos constitui um parâmetro de fácil monitoramento e capaz de detectar mudanças cristalinas em decorrência de variações no processo de síntese ou mesmo no processamento farmacêutico.

A distribuição granulométrica dos insumos exerce influência importante em aspectos relacionados às etapas da produção industrial de um medicamento, como em processos de mistura e de enchimento, assim como em sua estabilidade e eficácia biológica. No caso do BNZ, deve haver um controle rigoroso do tamanho de partícula, uma vez que este parâmetro físico possui influência direta em sua velocidade de dissolução, um fator crítico para sua biodisponibilidade. ${ }^{24}$

Os dados de tamisação do BNZ se ajustaram a uma distribuição logaritmo normal, o que permitiu determinar os valores de diâmetro médio de partícula e desvio padrão geométrico em 192,2 e 0,12 $\mu \mathrm{m}$, respectivamente. Conforme se observa no histograma da Figura 4, a distribuição do tamanho de partícula do BNZ é relativamente fechada e com tamanhos de partícula consideráveis.

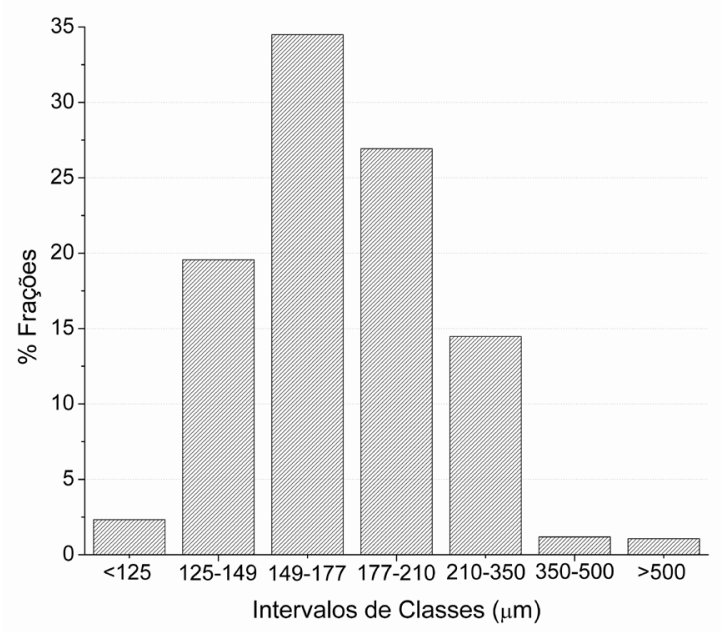

Figura 4. Distribuição de frequência do tamanho de partícula de BNZ por tamisação

\section{Propriedades de fluxo}

O estudo das propriedades de fluxo dos materiais é crucial no processo de fabricação de qualquer forma farmacêutica unidose, uma vez que exerce influência na alimentação uniforme das máquinas e, também, nas propriedades físicas e mecânicas dos comprimidos. ${ }^{25}$

Diferentemente de outros tipos de mensurações, a reologia dos sólidos não é uma propriedade inerente ao material e sim o resultado da combinação das propriedades físicas que afetam seu fluxo e do equipamento empregado no seu processamento, de maneira que é recomendável utilizar diferentes metodologias para avaliar este parâmetro; várias delas encontram-se descritas nas Farmacopeias. ${ }^{26}$

As medidas reológicas realizadas para a matéria-prima BNZ encontram-se resumidas na Tabela 3 e classificadas segundo Carr. ${ }^{13}$
Apesar de possuir um tamanho de partícula grande e de distribuição uniforme, o resultado dos diferentes ensaios realizados permite situar este fármaco como um material bastante coesivo e de mobilidade extremamente comprometida.

Tabela 3. Medidas de fluxo de BNZ. Entre parênteses está representado o desvio padrão dos resultados

\begin{tabular}{lcc}
\hline Parâmetro & Resultados & Classificação \\
\hline Ângulo de repouso $\left[^{\circ}\right]$ & $60(3,5)$ & Muito deficiente \\
Índice de compressibilidade $[\%]$ & $40,7(1,2)$ & Extremamente deficiente \\
Índice de Hausner & $1,68(0,03)$ & Extremamente deficiente \\
Tempo de escoamento $[\mathrm{s}]$ & $\infty$ & - \\
Densidade de enchimento $[\mathrm{g} / \mathrm{mL}]$ & $0,39(0,01)$ & - \\
\hline
\end{tabular}

O ângulo de repouso só pode ser medido após escoamento forçado do pó. As medidas de compressibilidade e de índice de Hausner confirmam o fluxo medíocre do material, apresentando valores muito superiores aos recomendados para insumos farmacêuticos.

O BNZ não flui livremente, possuindo tempo de escoamento infinito (Tabela 3 ). Este comportamento pode ser explicado pela morfologia das partículas (Figura $2 \mathrm{~S}$, material suplementar) que favorece interações interparticulares. A manipulação farmacêutica deste fármaco deve, portanto, requerer substâncias adjuvantes ou mesmo etapas adicionais de processamento industrial, que promovam a mobilidade de suas partículas.

O BNZ revela ainda um baixo valor de densidade de enchimento, o que praticamente o inviabiliza como candidato à compressão direta, método mais vantajoso de produção de comprimidos para a indústria farmacêutica. ${ }^{27}$

\section{Higroscopicidade}

A higroscopicidade de um fármaco refere-se a sua capacidade de captar água em função da umidade relativa ambiental. Considerando que a água é o veículo para as reações de decomposição dos princípios ativos, a estabilidade dos fármacos é fortemente afetada pelo seu percentual de umidade livre. As propriedades mecânicas dos materiais farmacêuticos também são criticamente influenciadas pelo teor de umidade dos sólidos. ${ }^{28}$

Os resultados deste estudo (Figura $3 \mathrm{~S}$, material suplementar) mostram que mesmo quando o BNZ é conservado em umidades relativas elevadas não há captação de água apreciável (inferior a $0,3 \%$ ), o que permite classificar este composto como um material não higroscópico. ${ }^{14} \mathrm{~A}$ umidade relativa atmosférica não representa, portanto, um parâmetro de risco para a manipulação e o armazenamento deste fármaco em estado sólido, de maneira que se poderão escolher materiais de envase mais flexíveis e baratos para este princípio ativo e suas formas farmacêuticas.

\section{CONCLUSÕES}

O estudo apresentado aporta importantes informações sobre a natureza físico-química do fármaco antichagásico benznidazol, em sua maioria inéditas, que ajudarão a nortear o melhoramento farmacotécnico do BNZ, assim como fornecem subsídios para o estabelecimento de um perfil de qualidade detalhado a ser adotado no controle de qualidade de rotina para este fármaco e para formulações que o utilizem como base.

Ao apresentar pela primeira vez sua classificação biofarmacêutica baseada em dados experimentais, será possível propor estratégias para minorar os problemas de biodisponibilidade deste fármaco, tentando suplantar não apenas sua limitada solubilidade aquosa, mas também 
utilizando ferramentas que possam melhorar a sua lipossolubilidade, através de adjuvantes farmacêuticos apropriados ou empregando sistemas de liberação vetorizados.

\section{MATERIAL SUPLEMENTAR}

Dados complementares estão disponíveis gratuitamente em http:// quimicanova.sbq.org.br, na forma de arquivo PDF. Neste material encontram-se perfil de dissolução intrínseca de BNZ (1S), fotomicrografia por MEV dos cristais de BNZ (2S), e isoterma de sorção do $\mathrm{BNZ}$ a $25^{\circ} \mathrm{C}(3 \mathrm{~S})$.

\section{AGRADECIMENTOS}

Este trabalho contou com o financiamento do CNPq projeto (472134/2008-6) e com o apoio das Universidades Federais de Ouro Preto (UFOP) e do Mato Grosso (UFMT). Os autores agradecem ao Laboratório Farmacêutico do Estado de Pernambuco (LAFEPE) em nome do Prof. J. L. Soares Sobrinho, da Universidade Federal do Piauí, pela doação da matéria-prima Benznidazol utilizada neste estudo.

\section{REFERÊNCIAS}

1. Joshi, H. N.; Int. J. Pharm. 2007, 343, 1.

2. Federsen, H. J.; Chirality 2003, 15, S128.

3. Wang, J.; Urban, L.; Drug Discovery World 2004, Fall, 73.

4. Pereira, A.; Bicalho, B.; Lilla, S.; De Nucci, G.; Quim. Nova 2005, 28, S107.

5. Swamivelmanickam, M.; Valliappan, K.; Reddy, P. G.; Madhukar, A.; Manavalan, R.; Int. J. ChemTech Res. 2009, 1, 1032.

6. Caldas, I. S.; Talvani, A.; Caldas, S.; Carneiro, C. M.; de Lana, M.; da Matta Guedes, P. M.; Bahia, M. T.; Parasitol. Res. 2008, 103, 413.

7. United States Pharmacopeia, $30^{\text {th }}$ ed., United States Pharmacopeial Convention: Rockville, 2008.

8. Zakeri-Milani, P.; Barzegar-Jalali, M.; Azimi, M.; Valizadeh, H.; Eur. J. Pharm. Biopharm. 2009, 73, 102.
9. OECD - Organisation for Economic Co-operation and Development; Guideline 123 - Partition coefficient n-octanol/water Slow-stirring method for highly hydrophobic chemicals, November 2003.

10. Stanley-Wood, N. G.; Enlargement and compaction of particulate solids. $1^{\text {st }}$ ed., Butterworths: London, 1983.

11. Fungaro, D. A.; Bruno, M.; Quim. Nova 2009, 32, 955.

12. Vila Jato, J.; Tecnología Farmacéutica, $1^{\text {st }}$ ed., Síntesis: Madrid, 1997.

13. Carr, R.; Chem. Eng. 1965, 72, 69.

14. Callahan, J. C.; Drug Dev. Ind. Pharm. 1982, 8, 355.

15. Greenspan, L.; J. Res. Nat. Bur. Stand. Sect. A. Phys. Chem. 1977, 81A, 89.

16. Lamas, M.; Villaggi, L.; Nocito, I.; Bassani, G.; Leonardi, D.; Pascutti, F.; Serra, E.; Salomon, C. J.; Int. J. Pharm. 2006, 307, 239.

17. Farmacopeia Brasileira, $4^{\mathrm{a}}$ ed., Atheneu: Rio de Janeiro, 1988.

18. Machatha, S. G.; Yalkowsky, S. H.; Int. J. Pharm. 2005, 294, 185.

19. Amidon, G. L.; Lennernas, H.; Shah, V. P.; Crison, J. R.; Pharm. Res. 1995, 12,413 .

20. Lima, A. A. N.; Soares-Sobrinho, J. L.; Silva, J. L.; Correa Júnior, R. A.; Lyra, M. A. M.; Rolim Neto, P. J.; Quim. Nova 2009, 32, 2196.

21. Kasim, N. A.; Whitehouse, M.; Ramachandran, C.; Bermejo, M.; Lennernas, H.; Hussain, A. S.; Junginger, H. E.; Stavchansky, S. A.; Midha, K. K.; Shah, V. P.; Amidon, G. L.; Mol. Pharm. 2004, 1, 85.

22. Bernal, C.; Couto, A. B.; Breviglieri, S. T.; Cavalheiro, E. T. G.; Quim. Nova 2002, 25, 849 .

23. Soares-Sobrinho, J. L.; Cunha-Filho, M. S. S.; Rolim Neto, P. J.; TorresLabandeira, J. J.; Dacunha-Marinho, B.; Acta Crystallogr., Sect. E: Struct. Rep. Online 2008, 64, o634.

24. Brandão, F. C. ; Tagiari, M. P.; Silva, A. S.; Berti, L. F.; Stulzer, H. K.; Pharm. Chem. J. 2008, 42, 368.

25. Schussele, A.; Bauer-Brandl, A.; Int. J. Pharm. 2003, 257, 301.

26. Prescott, J. K.; Barnum, R. A.; Pharm. Technol. 2000, 60.

27. Sá-Barreto, L. C. L.; Cunha-Filho, M. S. S.; Lat. Am. J. Pharm. 2009, $28,304$.

28. Aulton, M. E.; Pharmaceutics: The Design and Manufacture of Medicines, $3^{\text {rd }}$ ed., Elsevier: Oxford, 2007. 


\section{CARACTERIZAÇÃO FÍSICO-QUÍMICA DO FÁRMACO ANTICHAGÁSICO BENZNIDAZOL}

Flávia Pires Maximiano, Guilherme Hideki Yoshizane Costa e Jacqueline de Souza

Escola de Farmácia, Universidade Federal de Ouro Preto, Rua Costa Sena, 171, 35400-000 Ouro Preto - MG, Brasil

Marcílio Sérgio Soares da Cunha-Filho*

Instituto de Ciências da Saúde, Universidade Federal do Mato Grosso, Campus Universitário de Sinop, Av. Alexandre Ferronato, 1200, 78557-267 Sinop - MT, Brasil

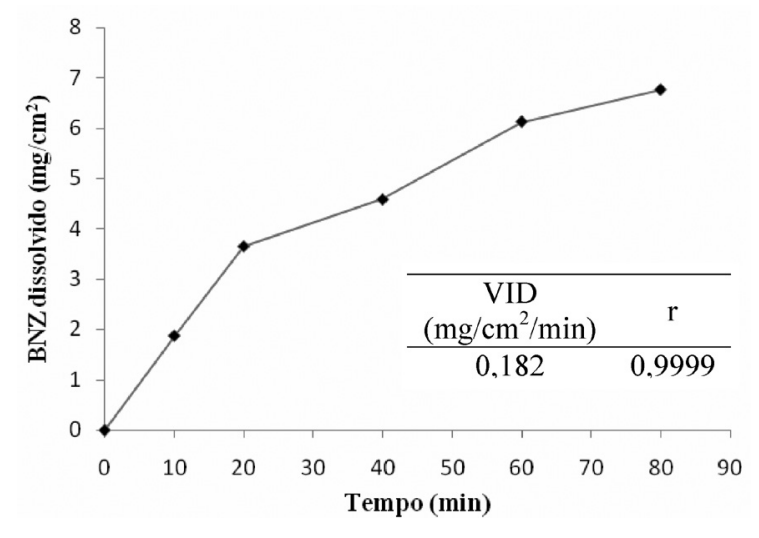

Figura 1S. Perfil médio de dissolução de BNZ, a partir de comprimidos de área superficial $1,13 \mathrm{~cm}^{2}$, em condições SINK a $37^{\circ} \mathrm{C}$, junto ao seu valor de velocidade intrínseca de dissolução (VID) e coeficiente de correlação linear (r) correspondente

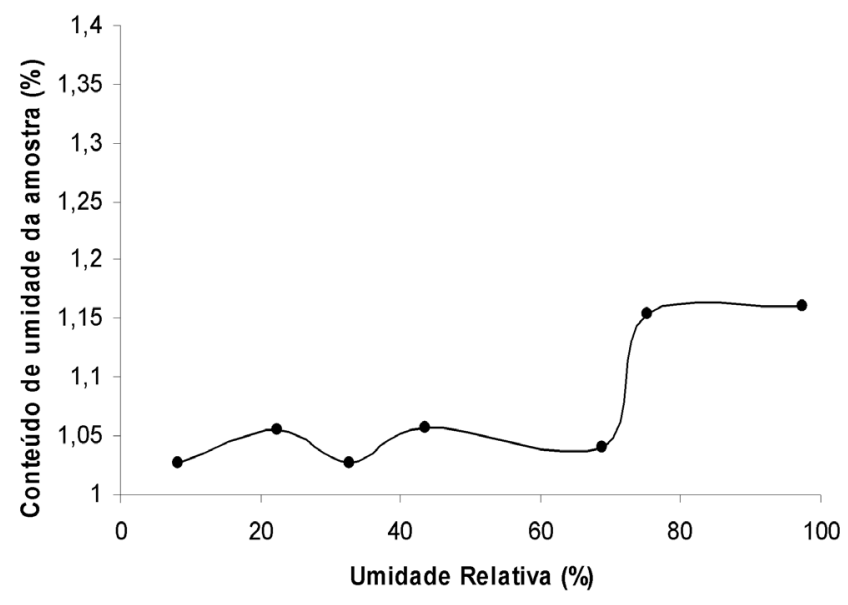

Figura 3S. Isoterma de sorção de umidade de BNZ a $25^{\circ} \mathrm{C}$

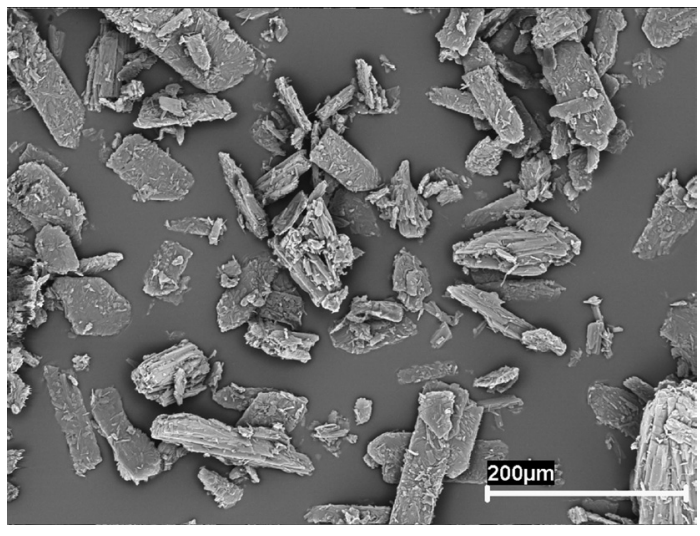

Figura 2S. Fotomicrografia por MEV de cristais típicos de BNZ 